\title{
EFFET DU POTASSIUM ET DU DEFICIT HYDRIQUE MODERE SUR LA TRANSPIRATION ET L'ABSORPTION POTASSIQUE CHEZ SIX GENOTYPES DE PALMIER AU STADE JUVENILE
}

\author{
L. NODICHAO', S. AKE ${ }^{2}$ et C. JOURDAN ${ }^{3}$ \\ ${ }^{1}$ Centre de Recherches Agricoles sur les Plantes Pérennes (CRA-PP), BP. 01 Pobè, Bénin \\ ${ }^{2}$ Université de Cocody-Abidjan, Laboratoire de Physiologie et Pathologie Végétales, UFR Biosciences, 22 B.P. 582 \\ Abidjan 22, Côte d'Ivoire. \\ ${ }^{3}$ CIRAD-CP, TA 80/01, 34398 Montpellier cedex 5
}

\begin{abstract}
RESUME
L'importance relative des ions $\mathrm{K}^{+}$et $\mathrm{Cl}^{-}$dans la tolérance du palmier à la sécheresse n'est pas complètement élucidée. De même, peu de données existent sur les interactions génotype x potassium $\mathrm{x}$ eau chez le palmier à huile. Le comportement de 6 génotypes de cette plante, sous deux régimes hydriques, avec ou sans apport de potassium, a été suivi dans des tubes en PVC. Le potassium a été apporté sous forme de $\mathrm{KCl}$ ou de $\mathrm{K}_{2} \mathrm{SO}_{4}$. II n'y a pas de différence entre les effets du $\mathrm{KCl}$ et du $\mathrm{K}_{2} \mathrm{SO}_{4}$ sur la transpiration par $\mathrm{mg}$ de feuilles sèches

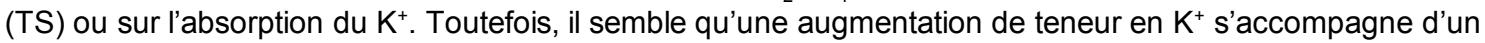
relèvement de la teneur en $\mathrm{Cl}^{-}$. Les différences de TS ou de teneur en potassium dans les feuilles ont varié selon le génotype, la dose de potassium et le régime hydrique.
\end{abstract}

Mots clés : Palmier à huile, tolérance à la sécheresse, transpiration, absorption potassique, absorption du chlore, Côte d'Ivoire

\author{
ABSTRACT \\ EFFECT OF POTASSIUM AND MIDDLE WATER DEFICIT ON RELATIVE TRANSPIRATION RATE AND POTASSIUM ABSORPTION \\ OF SIX GENOTYPES OF OIL PALM AT YOUNG STAGE
}

The relative importance of $\mathrm{K}^{+}$and $\mathrm{Cr}$ ions on palm oil tolerance to draught has yet to be elucidated. In addition, little is known about genotype $x$ potassium $x$ water interactions. An experiment was conducted to monitor the behaviour of 6 oil palm genotypes under two soil moisture regimes and $\mathrm{K}$ (as $\mathrm{K}_{2} \mathrm{SO}_{4}$ or $\mathrm{KCl}$ ) fertilisation. Results show no significant difference between Keffects on transpiration and $K$ absorption. However, It seems that the increase in foliar $\mathrm{K}^{+}$content was accompanied by a corresponding increase in foliar $\mathrm{Cl}^{-}$content. Differences between genotypes, in terms of transpiration and $K$ absorption, were found to be related to $K$ supply and soil water content.

Keywords : Oil palm, drought tolerance, transpiration, potassium absorption, chlorine absorption, Côte d'Ivoire

\section{INTRODUCTION}

L'engrais potassique souvent utilisé en élaeiculture est le chlorure de potassium $(\mathrm{KCl})$. Pour une production moyenne de $15 \mathrm{t} / \mathrm{ha}$, les besoins en $\mathrm{N}$ et $\mathrm{K}$ sont identiques et s'élèvent à $90 \mathrm{~kg} / \mathrm{ha}$, contre un besoin de $15 \mathrm{~kg} /$ ha de $\mathrm{P}$
(Quencez et De Taffin, 1981). Malgré ce niveau élévé de $K$ dans cette plante, l'importance relative des ions $\mathrm{K}^{+}$et $\mathrm{Cl}^{-}$est souvent discutée. Dans une expérience de fumure minérale au palmier, Ollagnier et Ochs (1971) ont attribué les gains de productions obtenus grâce à des apports de $\mathrm{KCl}$, aux ions $\mathrm{Cl}^{-}$plutôt qu'aux ions $\mathrm{K}^{+}$. Mais, Ollagnier (1985) trouve que le palmier à huile 
est beaucoup moins sensible à une carence en chlore que le cocotier, une autre palmacée. De Taffin et Ochs (1973) préfèrent l'utilisation du sulfate de potassium au chlorure de potassium quand la teneur en ions $\mathrm{Cl}^{-}$dépasse $0,6 \%$ dans les feuilles. Les ions $\mathrm{K}^{+}$sont impliqués dans plusieurs processus physiologiques importants, et contribuent à l'augmentation de la résistance à la sécheresse (Soltner, 1994).

Cependant, Bonneau (1998) a confirmé chez le cocotier, que l'élément nutritif le plus important à la tolérance à la sécheresse est l'ion $\mathrm{Cl}^{-}$. La carence en ion $\mathrm{Cl}^{-}$provoque une baisse de conductance stomatique chez le cocotier (Braconnier et d'Auzac, 1985). Celle-ci entraine donc une chute de transpiration, et explique en partie, les baisses de production en situations de déficience en chlore. La carence en ions $\mathrm{Cl}^{-}$ entraînerait une réduction de disponibilité en osmoticums en cas de stress hydrique (Braconnier, 1988), et peut donc avoir une conséquence négative sur la tolérance à la sécheresse.

Il a été rapporté, chez le palmier à huile, que certains génotypes sensibles conservent pendant la saison sèche, leurs stomates ouverts plus longtemps que ceux des génotypes tolérants (Cornaire et al., 1994). Les différences de comportements stomatiques entre génotypes sensibles et tolérants peuvent expliquer par les différences de teneur foliaire en ions $\mathrm{Cl}^{-}$ou $\mathrm{K}^{+}$, car il est montré que des écarts existent entre génotypes de palmier à huile pour le développement du système racinaire (Cornaire et al., 1994) qui constitue le principal organe de mobilisation de l'eau et des minéraux chez les plantes.

Ce travail vise la caractérisation de la sensibilité de six génotypes de palmier à huile vis-à-vis des déficits hydrique et potassique d'une part, et d'autre part, à élucider l'importance relative des ions $\mathrm{K}^{+}$et $\mathrm{Cl}^{-}$pour la tolérance du palmier à huile à la sécheresse.

\section{MATERIEL ET METHODES}

\section{MATERIEL VEGETAL}

Six génotypes de palmier à huile ont été utilisés pour cette expérimentation. Les semences ont été obtenues auprès du Centre de Recherche Agricole sur les Plantes Pérennes du Bénin (CRA-PP). La tolérance présumée des génotypes à la sécheresse et l'origine des parents, sont consignées dans le tableau 1.

Tableau 1 : Tolérance présumée de six génotypes de palmier à huile à la sécheresse selon leur catégorie et l'origine des parents

Presume resistance of six oil palm genotypes to drought according to category and parents.

\begin{tabular}{llcccc}
\hline Catégorie & Origine & \multicolumn{2}{c}{ Génotypes } & $\begin{array}{l}\text { Tolérance } \\
\text { présumée }\end{array}$ & Code \\
& Dura $x$ Ténéra & femelle & Mâle & & \\
C1401 & DA10DxLM2T & PO3719D & PO4102P & Intermédiaire & A \\
C1001F & DA115DxLM2T & PO3179D & PO2558P & Intermédiaire & B \\
C6446 & DA8Dx(LM9TxLM13T) & PO3724D & PO3643P & Tolérant & C \\
C2101 & (DA10DxDA3D)xLM2T & PO2544D & PO2558P & Inconnue & D \\
C2001 & (LM404DxDA10D)xLM2T & PO4672D & PO2558P & Sensible & E \\
C2501 & (DA3DxDA5D)xLM2T & PO5251D & PO4102P & Inconnue & F \\
\hline
\end{tabular}




\section{METHODES}

\section{Culture en prépépinière}

La mise en germination des graines a été effectuée suivant la méthode standard de l'IRHO (Corrado et Wuidart, 1990). Des graines germées de 1,5 à $2 \mathrm{~cm}$ de longueur radiculaire ont été triées et semées dans de petits pots de polyéthylène de $20 \mathrm{~cm}$ de profondeur $x 8,5 \mathrm{~cm}$ de diamètre. Chaque pot a été rempli de terreau jusqu'à $3 \mathrm{~cm}$ de son bord. Les plantules ont été placées sous ombrage pendant toute la phase de prépépinière. Elles ont été arrosées chaque matin entre $7 \mathrm{~h} 30$ et $8 \mathrm{~h}$ pendant 50 à 60 jours.

\section{Transfert dans les tubes PVC}

Les plantules ont été transférées dans des tubes au polyvinyle (PVC) de $95 \mathrm{~cm}$ de long et $10 \mathrm{~cm}$ de diamètre. Au moment du transfert, la hauteur, le diamètre du pseudobulbe et le nombre de feuilles des plantules ont été mesurés et des plants de taille similaire retenus. Chaque plantule a été délicatement débarrassée de sa motte de terre, en déchirant le pot à la base, et en lavant les racines dans un seau d'eau. Les plantules ont été transférées dans des tubes PVC contenant chacun $9 \mathrm{~kg}$ de sol tassé à une densité de 1,2 à $1,3 \mathrm{~g} \mathrm{~cm}^{-3}$. Après le transfert de la plantule, le sol a été immédiatement saturé d'eau et soumis à un drainage libre pendant 48 h. La surface du sol a été protégée par une toile plastique en polyéthylène noire afin de minimiser les pertes d'eau par évaporation. L'eau drainée a été recueillie dans de petits bols plastiques placés sous les tubes et réutilisée au besoin. Un arrosage à l'eau (quotidien) équivalent à l'évapotranspiration a été assuré par tube jusqu'à la mise en place d'un régime de déficit hydrique.

\section{Traitements}

\section{Application des traitements potassiques}

Trois traitements potassiques (K0, K1, K2) ont été appliqués. A cet effet, trois solutions minérales $\mathrm{K} 0, \mathrm{~K} 1$ et $\mathrm{K} 2$ aux concentrations respectives de $0 \mathrm{~g}, 2.10^{-3} \mathrm{~g}$ et $3.10^{-3} \mathrm{~g}$, ont été apportées au sol. Les doses de l'ensemble des éléments minéraux, sont présentées dans le tableau 2. Le sol utilisé est un sol ferrallitique prélevé dans les $25 \mathrm{~cm}$ supérieurs dans un ancien champ de palmier à huile. La teneur initiale du sol en $\mathrm{K}$ a été de 0,16 méq / $100 \mathrm{~g}$. Le sol collecté a été réparti en trois lots et chaque lot a été humidifié avec une des trois solutions. Le sol ainsi humidifié a été homogénéisé avec une petite bétonnière motorisée, puis séché sous abri.

Tableau 2 : Quantité de sels minéraux utilisée par tube selon les traitements potassiques.

Rates of minerals used per tube according to potassium treatments.

\begin{tabular}{lccc}
\hline \multirow{2}{*}{ Sel minéral $(\mathrm{g})$} & $\begin{array}{c}\text { Traitement } \\
\text { sans K }(\mathrm{g})\end{array}$ & \multicolumn{2}{c}{ Traitement avec K } \\
\cline { 2 - 4 } & $(\mathrm{K} 0)$ & $\begin{array}{c}\text { Sous forme de } \mathrm{KCl}(\mathrm{g}) \\
(\mathrm{K} 1)\end{array}$ & $\begin{array}{c}\text { Sous forme de } \mathrm{K}_{2} \mathrm{SO}_{4}(\mathrm{~g}) \\
(\mathrm{K} 2)\end{array}$ \\
\hline Urée & 0,008 & 0,008 & 0,008 \\
Diammonium hydrogénophosphate & 0,003 & 0,003 & 0,003 \\
Chlorure de potassium & 0 & 0,002 & 0 \\
Sulfate de potassium & 0 & 0 & 0,003 \\
\hline
\end{tabular}

\section{Déficit hydrique}

Après $75 \mathrm{j}$ de culture en l'absence de stress hydrique, la moitié des plantules a été soumise à un régime de déficit hydrique progressif pendant $16 \mathrm{j}$. Le déficit hydrique quotidien a été calculé suivant la méthode des pesées successives (Sinclair et Ludlow, 1986). Les plantules, sous régime de déficit hydrique, ont reçu, par jour, une quantité d'eau équivalente à la perte du poids de leur tube au bout de $24 \mathrm{~h}$ moins la quantité d'eau minimale perdue par évapotranspiration relevée sur l'ensemble des tubes du jour. Chaque plantule, en régime normal, a été quotidiennement arrosée avec une quantité d'eau égale à l'évapotranspiration en $24 \mathrm{~h}$. La balance utilisée, pour quantifier la perte d'eau des tubes, a une portée de $35 \mathrm{~kg}$. Elle a été étalonnée pour une erreur de $2 \mathrm{~g}$. Au bout 
des $16 \mathrm{j}$ suivant la réduction d'arrosage, la réserve d'eau utilisable $(\mathrm{RU})$ dans les tubes, sous régime de déficit hydrique a été de $77 \%$ de la réserve d'eau maximale utilisable ( $1 \mathrm{RU})$ dans les tubes sous régime hydrique normal. Les résultats correspondent à deux régimes hydriques ; $1 \mathrm{RU}$ (normal) et un dessèchement progressif qui a atteint une humidité de $3 / 4 \mathrm{RU}$ (un faible déficit hydrique) à la fin de l'essai.

Afin de réduire l'impact de la taille des plants sur les résultats, la transpiration spécifique (TS) a été calculée. Elle a été déterminée en divisant la transpiration par pot, par la biomasse foliaire correspondante. Celle-ci a été mesurée au lendemain $\mathrm{du} 16^{\mathrm{e}} \mathrm{j}$ du régime de déficit hydrique à la fin de l'essai.

\section{Dispositif expérimental et mesures}

Un essai factoriel 6 (génotypes) $\times 3$ (traitements potassiques) $\times 2$ (régimes hydriques), a été utilisé suivant un plan en bloc aléatoire complet avec quatre répétitions. Au total 144 plantules ont été transférées, à raison de 24 par génotype. Chaque jour, la transpiration des plantules est mesurée à 16 h 30 suivant la technique des pesées successives décrites plus haut, en supposant que l'évaporation du sol est négligeable. La hauteur des plantules est mesurée une fois par semaine. La technique utilisée consiste à regrouper l'ensemble des feuilles et à prendre la hauteur de la feuille la plus longue. Le nombre de feuilles est aussi noté tous les 7 jours, à partir du transfert des plantules jusqu'à la fin de l'essai. Au dernier jour de l'essai, les plants ont été délicatement retirés des tubes sous une faible pression d'eau, pour débarrasser les racines des particules de sol. La longueur maximale des racines primaires a été mesurée à l'aide d'une règle et la surface totale calculée à l'aide du logiciel WINRHIZO de Régent Instruments Inc. Version $4.1 \mathrm{c}$, à partir d'images racinaires scannées avec un scanner EPSON 1600.

Le diamètre du pseudobulbe a été mesuré à l'aide d'un pied à coulisse ordinaire. La matière sèche des feuilles a été déterminée après passage à l'étuve à $105^{\circ} \mathrm{C}$ pendant $48 \mathrm{~h}$. Des échantillons de feuilles séchées ont été soumis à des analyses pour $\mathrm{Cl}, \mathrm{S}$ et $\mathrm{K}$ au $\mathrm{CIRAD}$ à Montpellier.

Les résultats présentés concernent les effets des traitements hydropotassiques sur la transpiration et la teneur en minéraux dans les feuilles des génotypes de palmier à huile.

\section{Analyses des données}

Les effets des traitements ont été déterminés par analyse de variance, avec le logiciel GenStat (version 4.2). Les sources de variations ont été décomposées en génotype, traitement potassique et régime hydrique comme effets principaux, ainsi que leurs interactions. La comparaison des moyennes a été faite selon le test de la plus petite différence significative (PPDS) aux seuils de 5 $\%$ ou $1 \%$, à l'aide des erreur standard.

\section{RESULTATS}

\section{TENEUR EN K, CI ET S DES FEUILLES}

Le tableau 3 présente les quantités totales de $K$ accumulées dans les feuilles, en fonction du traitement potassique. L'apport des deux types d'engrais potassiques ( $K 1$ et $K 2$ ) a favorisé un plus grand prélèvement de $\mathrm{K}$ par la plante pour tous les génotypes. En revanche, il n'y a pas eu de différence entre les effets des deux types d'engrais. Le génotype $D$ a de façon significative (seuil $5 \%$ ) prélevé plus d'ions $\mathrm{K}^{+}$que les génotypes $A$ et $E$.

La réduction de l'humidité du sol a entrainé une baisse de la teneur moyenne (génotypes) au K dans les feuilles, sauf chez les plantules traitées avec K1 (Figure 1). Toutefois, les variations entre les différents traitements n'ont pas été significatives. L'augmentation de la teneur en ions chlore ou sulfate a été significative dans les tubes ayant reçu $\mathrm{KCl}$ ou $\mathrm{K}_{2} \mathrm{SO}_{4}$ seulement (Figures 2 et 3).

Comme nous n'avons pas pu montrer l'existence d'une différence significative entre $\mathrm{KCl}_{\text {et }} \mathrm{K}_{2} \mathrm{SO}_{4}$, pour leur effet sur l'accumulation de K (Tableau 3 et Figure 1), l'effet des deux types d'engrais a été confondu pour la teneur en minéraux chez les génotypes. $\mathrm{K}^{+}$est l'ensemble des traitements $\mathrm{K} 1$ et K2. Les variations entre plantules (Tableau 4) pour le même traitement, (combinaison génotype, traitement potassique et régime hydrique), de la teneur de $\mathrm{K}$, n'ont pas permis d'établir une interaction significative (Tableau 4). II apparaît cependant, que les teneurs en ions $\mathrm{K}^{+}$ont varié entre génotypes, selon selon le traitement potassique K et l'humidité du sol (Tableau 4). 


\section{TRANSPIRATION}

La figure 4 montre la variation de la transpiration spécifique (TS) moyenne de tous les génotypes selon le régime hydrique et le traitement potassique au $16^{\mathrm{e}}$ jour de déficit hydrique. Le régime $3 / 4 R U$ a réduit la $T S$, quelque soit l'apport potassique. La différence entre les traitements potassiques n'a pas été significative. La différence entre les effets de $\mathrm{KCl}$ et de $\mathrm{K}_{2} \mathrm{SO}_{4}$ sur la TS, n'ayant pas été significative, les effets des deux types d'engrais ont été confondus pour comparer la TS chez les génotypes. La différence entre génotypes a varié selon la dose d'engrais potassique et le régime hydrique (Tableau 5).

Tableau 3 : Quantité totale de K (mg / plant) accumulée dans les feuilles de six génotypes de palmier à huile suivant les traitements potassiques

Quantity of total K (mg / plant) absorbed by six oil palm genotypes according to potassium treatments.

\begin{tabular}{lcccc}
\hline \multirow{2}{*}{ Génotypes $(\mathrm{G})$} & \multicolumn{3}{c}{ Traitements potassiques $(\mathrm{K})$} & \multirow{2}{*}{ Moyenne (génotypes) } \\
\cline { 2 - 4 } & $\mathrm{K} 0$ (témoin) & $\mathrm{K} 1(\mathrm{KCl}$ & $\mathrm{K} 2\left(\mathrm{~K}_{2} \mathrm{SO}_{4}\right)$ & $18,6^{*}$ \\
$\mathrm{~A}$ & 14,4 & 19,4 & 24,6 & 19,6 \\
$\mathrm{~B}$ & 16,8 & 21,2 & 22,8 & 21,1 \\
$\mathrm{C}$ & 16,9 & 24,8 & 24,6 & 25,8 \\
$\mathrm{D}$ & 22,8 & 31,1 & 26,0 & $18,9^{*}$ \\
$\mathrm{E}$ & 16,3 & 19,1 & 22,9 & 23,3 \\
$\mathrm{~F}$ & 20,0 & 28,6 & 24,0 & 21,3 \\
\hline Moyenne $(\mathrm{K})$ & $18,0^{* *}$ & 24,3 & 24,2 & \\
\hline
\end{tabular}

$\mathrm{L}^{\prime}$ Interaction $\mathrm{G} \times \mathrm{K}$ n'est pas significative ; l'erreur standard $=4,9 \mathrm{mg}$.

$\mathrm{G} \times \mathrm{K}$ interaction is not significant ; standard error is $4,9 \mathrm{mg}$

${ }^{*}=$ différence significative au seuil de $5 \% ;{ }^{* *}=$ différence significative au seuil de $1 \%$.

${ }^{*}=$ significant difference at $5 \% ;{ }^{* *}=$ significant difference at $1 \%$.

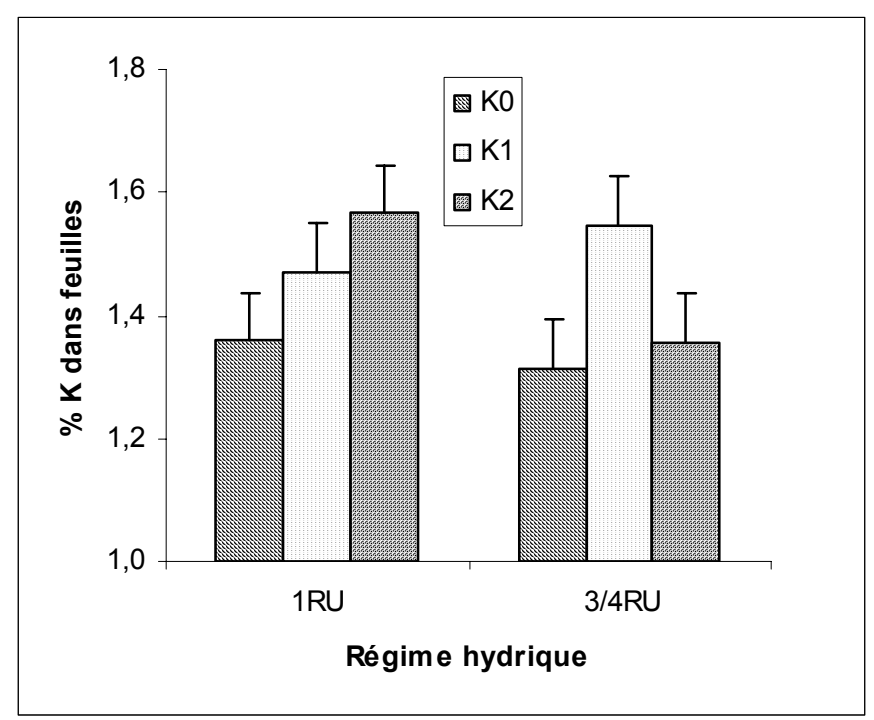

Figure 1 : Variation de la teneur en $\mathrm{K}$ dans les feuilles de palmier à huile selon les traitements potassiques et le régime hydrique. Les barres représentent l'erreur standard.

Oil palm foliar $K$ content (\%) variation according to $K$ treatments and soil water regimes. Bars represent standard error. 


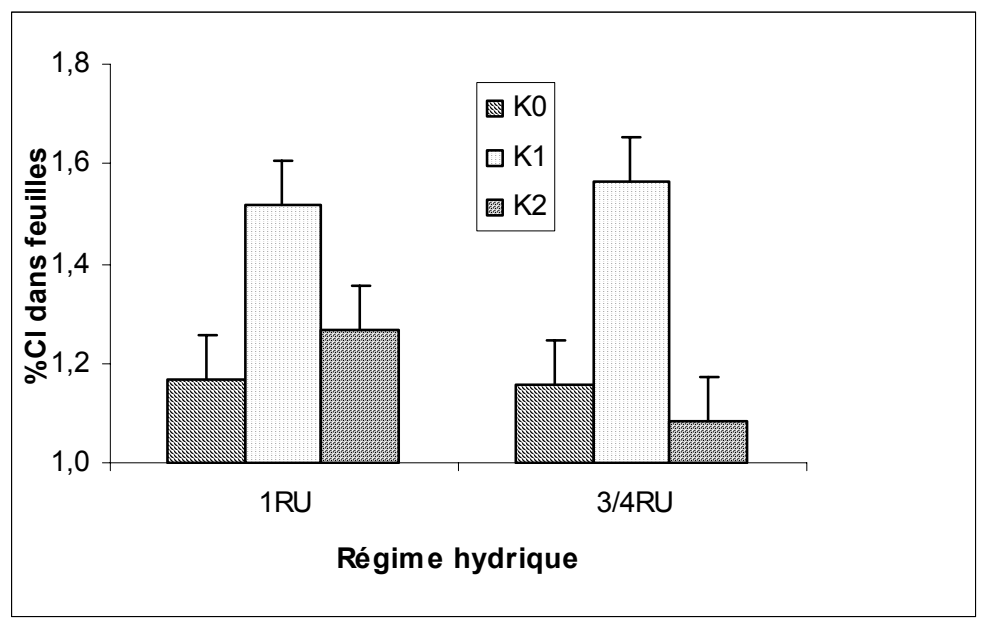

Figure 2 : Variation de la teneur en $\mathrm{Cl}(\%)$ des feuilles de palmier à huile selon les traitements potassiques et le régime hydrique.

Changes in $\mathrm{Cl}(\%)$ contents of palm oil leaves as a function of $\mathrm{K}$ treatments and soil water regimes.

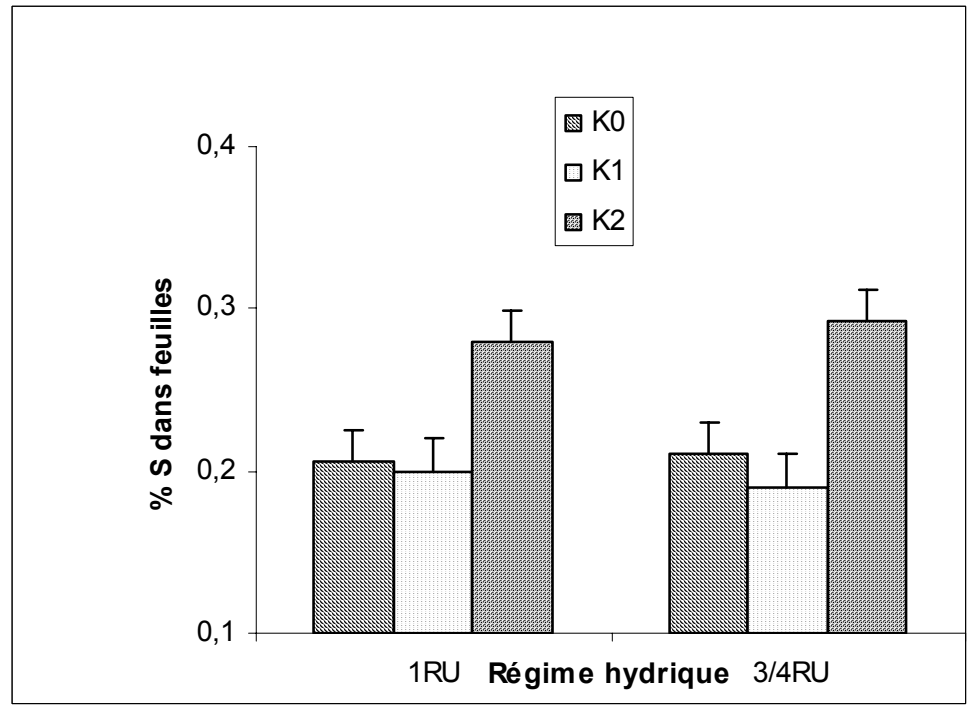

Figure 3 : Variation de la teneur en $S(\%)$ des feuilles de palmier à huile selon les traitements potassiques et le régime hydrique.

Changes in sulphur content (\%) of palm-oil leaves according to $K$ treatments and soil water regimes. 
Tableau 4 : Teneur en potassium (\%) des feuilles de six génotypes de palmier à huile selon le traitement potassique et le régime hydrique.

Foliar potassium content (\%) of six oil palm genotypes according to potassium treatments and water regimes.

\begin{tabular}{|c|c|c|c|c|c|}
\hline \multirow{2}{*}{ Génotypes } & \multicolumn{2}{|c|}{$1 R U$} & \multicolumn{2}{|c|}{$3 / 4 R U$} & \multirow{2}{*}{$\begin{array}{l}\text { Moyenne } \\
\text { génotypes }\end{array}$} \\
\hline & $+\mathrm{K} 0$ & $+K$ & $+\mathrm{K} 0$ & $+\mathrm{K}$ & \\
\hline$A$ & 1,4 & 1,7 & 1,7 & 1,4 & 1,5 \\
\hline B & 1,5 & 1,3 & 1,3 & 1,4 & 1,4 \\
\hline C & 1,5 & 1,7 & 1,3 & 1,4 & 1,5 \\
\hline$D$ & 1,2 & 1,6 & 1,1 & 1,4 & 1,3 \\
\hline$E$ & 1,3 & 1,4 & 1,2 & 1,6 & 1,4 \\
\hline $\mathrm{F}$ & 1,3 & 1,5 & 1,3 & 1,6 & 1,4 \\
\hline Moyenne & 1,4 & $1,5^{*}$ & 1,3 & $1,5^{*}$ & \\
\hline
\end{tabular}

Interaction génotype $x$ potassium $x$ eau est non significative, l'erreur standard $=0,2 \%$.

* significatif au seuil $5 \%$ de PPDS.

Interaction génotype $x$ potassium $x$ water is not significant, standard error $=0,2 \%$.

* significant at $5 \%$ de LDS.

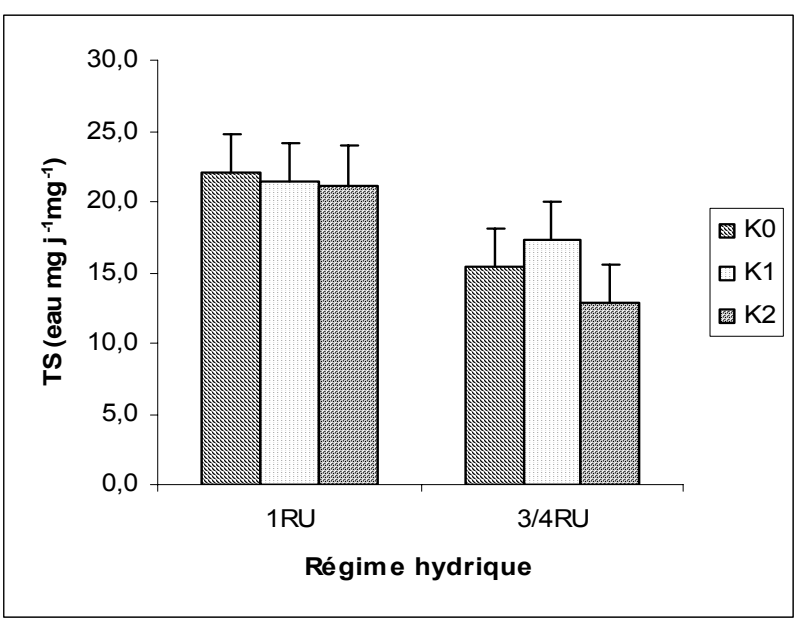

Figure 4 : Transpiration spécifique ( $m g$ eau j-1 mg -1MS) selon le régime hydrique et les traitements potassiques chez le palmier à huile.

Specific transpiration of foliar dry matter of oil palm at two water regimes and potassium treatments.

Tableau 5 : Transpiration spécifique $\left(\mathrm{mg}\right.$ eau $\left.\mathrm{j}^{-1} \mathrm{mg}^{-1} \mathrm{MS}\right)$ selon deux régimes hydriques et deux niveaux potassiques chez six génotypes de palmier à huile.

Specific transpiration of six oil palm genotypes according to soil water regimes and potassium treatments .

\begin{tabular}{lrrrr}
\hline \multirow{2}{*}{ Génotype } & \multicolumn{2}{c}{$\mathrm{K} 0$} & $\mathrm{~K} U$ & $3 / 4 \mathrm{RU}$ \\
\cline { 2 - 5 } & $\mathrm{RU}$ & $3 / 4 \mathrm{RU}$ & 27,3 & $14,4^{*}$ \\
$\mathrm{~A}$ & 27,9 & 21,6 & 16,1 & 12,7 \\
$\mathrm{~B}$ & 26,0 & $12,6^{*}$ & 25,1 & 15,7 \\
$\mathrm{C}$ & 20,9 & 21,0 & 20,5 & 11,3 \\
$\mathrm{D}$ & 15,0 & 14,8 & 21,2 & 18,8 \\
$\mathrm{~F}$ & 21,7 & $7,9^{*}$ & 18,0 & 18,2 \\
\hline Moyenne & 22,8 & 15,9 & 21,4 & $15,3^{*}$ \\
\hline
\end{tabular}

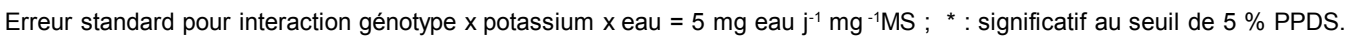

Interaction $\mathrm{x}$ potassium $\mathrm{x}$ water is not significant, standard error $=0.2 \% ;{ }^{*}=$ significant at $5 \%$ LDS. 


\section{DISCUSSION}

\section{IMPORTANCE RELATIVE DU K ET DE CI SUR LATOLERANCE AU DEFICIT HYDRIQUE}

Dans les tubes sous régime hydrique déficitaire (3/4RU), les variations de teneur en $\mathrm{K}^{+}$ou en $\mathrm{Cl}^{-}$ dans les feuilles sont accompagnées de variation de TS dans le même sens surtout pour $\mathrm{Cl}$. En effet, l'apport de $\mathrm{KCl}$ a permis d'améliorer sous déficit hydrique la teneur moyenne en $\mathrm{Cl}$ (tous génotypes) de $30 \%$ par rapport aux plantules traitées avec du $\mathrm{K}_{2} \mathrm{SO}_{4}$. Parallèlement la TS a augmenté dans une proportion du même ordre (31\%). De plus, cette augmentation de teneur en chlore a été aussi suivie d'une plus grande absorption de $\mathrm{K}^{+}(9 \%)$ en faveur des plantules traitées avec du KCl. Par contre, l'augmentation de teneur en $S$ n'a pas été suivie d'un supplément de TS. Ces résultats montrent que les ions $\mathrm{K}^{+}$et $\mathrm{Cl}^{-}$sont importants pour le maintien de la transpiration du palmier à huile sous déficit hydrique, au moins pour un niveau de déficit hydrique de l'ordre de celui appliqué dans cette étude.

ASPECTS PHYSIOLOGIQUES DE L'IMPORTANCE DU KET DE CI SUR LATOLERANCE AU DEFICIT HYDRIQUE

D'un point de vue physiologique, une plus grande transpiration suppose une plus grande conductance stomatique qui devrait se traduire par une plus grande mobilisation d'ions $\mathrm{K}^{+}$et

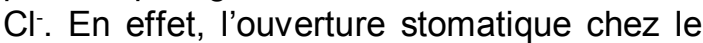
palmier à huile et chez le cocotier s'accompagne d'un mouvement de $\mathrm{K}^{+}$et $\mathrm{Cl}^{-}$des cellules subsidiaires latérales vers les cellules de garde (Braconnier et d'Auzac, 1985). La teneur en ions $\mathrm{K}^{+}$dans les cellules de garde augmente de 50 $\%$, tandis que celle des ions $\mathrm{Cl}^{-}$double presque (Braconnier, 1988). Il est montré chez le cocotier que les ions $\mathrm{Cl}$ - permettent d'une part, de garder les stomates ouverts plus longtemps dans la journée, et d'autre part, de maintenir la turgescence des cellules grâce à un abaissement du potentiel hydrique (Braconnier et d'Auzac, 1989).

Cependant, malgré les fortes teneurs en $\mathrm{Cl}$ et en $S$, il n'a pas été possible d'établir une différence significative entre les trois traitements $\mathrm{KO}+3 / 4 \mathrm{RU}$ $\mathrm{K} 1+3 / 4 \mathrm{RU}$ et $\mathrm{K} 2+3 / 4 \mathrm{RU}$ pour la transpiration spécifique. Ces résultats permettent de dire que la TS était plus conditionnée par la concentration en $\mathrm{K}^{+}$dans les feuilles, qui, dans les conditions de l'expérience, a beaucoup varié entre plantules de même traitement. L'absence de différence significative entre les trois traitements potassiques suggère que la teneur en $\mathrm{K}^{+}$dans les feuilles avant l'application du déficit hydrique était, suffisante pour assurer une transpiration normale chez les plantules dans les trois traitements. Ceci se confirme dans les tubes sous régime hydrique normal ( $1 \mathrm{RU})$, où malgré, des variations de teneur en $\mathrm{K}^{+}$entre les traitements +K0+1RU, +K1+1RU et +K2+1RU la TS était pratiquement identique, avec une légère augmentation chez les plantules du traitement +K0+1RU.

II est possible cependant, que cette augmentation non significative soit tout simplement un artefact lié à l'hétérogénéité dans les tailles des plantules, plutôt qu'un réel effet lié au traitement. Néanmoins, Bonneau (1998), a aussi observé en régime sans déficit hydrique, des jeunes plantules de cocotier à faible teneur en $\mathrm{Cl}$, se comporter mieux que des plantules dont la teneur en $\mathrm{Cl}$ était significativement plus élevée. Mais il a remarqué que ce comportement s'inversait sous déficit hydrique. Les effets dépressifs du $\mathrm{Cl}$ en conditions d'humidité du sol non- limitantes, et positif dans les situations de déficit hydrique ont été également observées chez le blé noir par Kozlova (1973) cité par Bonneau (1998). L'éventualité d'un effet dépressif du Cl n'est donc pas à écarter.

\section{INFLUENCE DE LA TENEUR INITIALE DU SOL}

L'absence de différence significative entre les traitements, pour ce qui concerne l'effet du chlore sur la transpiration, peut s'expliquer par les teneurs assez élevées de $\mathrm{Cl}(>1 \%)$ dans les feuilles des plantules des traitements sans apport de $\mathrm{Cl}$. Cela indique que le sol utilisé pour la préparation des mélanges de sol, n'était pas carencé en $\mathrm{Cl}$. Cependant, nos résultats justifient l'utilisation de $\mathrm{KCl}$ sur ce type de sol, car, même si le supplément de transpiration lié au $\mathrm{KCl}$ dans les tubes, sous régime hydrique déficitaire, n'était pas significatif, il n'est pas négligeable et est cohérent avec l'effet bénéfique du $\mathrm{Cl}$ sur la tolérance des palmacées à la sécheresse (Braconnier, 1988 ; Ollagnier, 1985). Toutefois, nos résultats montrent aussi que, dans les situations de non carence en Cl l'impact du $\mathrm{Cl}$ est moins marqué sur la transpiration du palmier à huile et peut être insignifiant quand I'humidité du sol est suffisante. 
L'absence de différence significative entre les effets des traitements avec le $\mathrm{K}_{2} \mathrm{SO}_{4}$ et le $\mathrm{KCl}$, suggère que ces deux types d'engrais pourraient être employés sur le palmier sur un sol similaire à celui utilisé dans ce travail. Ce résultat semble donc soutenir l'utilisation de sulfate de potassium au lieu du chlorure de potassium lorsque la teneur en chlorure dans les tissus foliaires dépasse 0,6\% (De Taffin et Ochs, 1973). Mais on pourrait craindre une acidification du sol due à une utilisation continue de l'engrais sulfaté. Cependant, le risque d'un effet dépressif du KCl sur la production du palmier à huile est également signalé par De Taffin et Ochs (1973). II serait donc logique d'étudier l'effet du KCl dans les interactions génotype $\mathrm{x}$ potassium $\mathrm{x}$ eau, pour en fixer les doses optimales selon les génotypes et les conditions de culture.

\section{INTERACTION GENOTYPE X POTASSIUM X HUMIDITE DU SOL}

Quoiqu'on ne puisse pas établir des différences significatives entre les traitements, pour la teneur en $\mathrm{K}$ dans les feuilles, les résultats en rapport avec la capacité au champ, montrent que les génotypes $E$ et $B$ ont accumulé moins de $K$ dans les feuilles que les autres génotypes, notamment dans les tubes qui ont reçu un apport d'engrais potassique. Pour les autres conditions, la différence entre les autres génotypes et le génotype $E$, a varié selon le cas.

Les variations de teneur en $\mathrm{K}^{+}$ont été accompagnées de variations de $\mathrm{Cl}$ dans la plupart des cas, et concordent avec les variations de la transpiration. Cependant, il est apparu que le potassium apporté sous le régime hydrique limitant, a été utilisée diversement par les génotypes. Chez le génotype $E$ l'apport du $K$ sous le régime hydrique limitant, a été utilisé vraisemblablement comme osmoticum au cours du dessèchement du sol avec pour impact, une plus grande accumulation de $\mathrm{K}$, et un maintien de la TS. La sensibilité présumée à la sécheresse du génotype $\mathrm{E}$ ne serait donc pas liée à une incapacité d'osmorégulation. Le $\mathrm{K}^{+}$apporté au génotype $\mathrm{C}$ a dû jouer à la fois un rôle nutritionnel et régulateur. II est possible que la tolérance du génotype $C$ à la sécheresse, résulte d'une capacité d'optimiser l'équilibre entre la transpiration et l'assimilation (Braconnier et d'Auzac, 1989).

\section{INFLUENCE POSSIBLE DE STRESS AUTRES QUE LE DEFICIT HYDRIQUE}

Le déficit hydrique lié à une réserve hydrique de $3 / 4 R U$ ne permet pas d'expliquer la réduction de transpiration observée chez les plantules notamment chez le génotype $E$ sous le traitement $+K 0+3 / 4 R U$ (63 \% TS). L'effet du déficit hydrique a dû être aggravé par d'autres stress, en particulier le stress thermique. En effet, lors des expérimentations, une forte chaleur et une période d'harmattan inattendue ont été observées. Les températures sous serre dépassaient de 4 à $6{ }^{\circ} \mathrm{C}$ dans la journée, les températures ambiantes relevées au poste météo du CRA-PP où l'étude a été menée. Durant les mesures, les températures maxima et minima enregistrées au Centre, avaient varié entre $25,3-34,5^{\circ} \mathrm{C}$, et $20,6-25^{\circ} \mathrm{C}$. Toutefois, l'influence de ces facteurs non contrôlés n'invalide pas les résultats, car elle est la même pour tous les traitements étudiés. Mais le niveau de déficit hydrique $(3 / 4 R U)$ est encore très modéré pour induire des effets d'interaction génotype $x$ dose de $K x$ régime hydrique significatifs chez les génotypes.

\section{CONCLUSION}

Malgré la plus grande accumulation de $\mathrm{Cl}$ chez les plantules ayant reçu du $\mathrm{KCl}$ par rapport à celles qui ont été traitées avec du $\mathrm{K}_{2} \mathrm{SO}_{4}$, nous n'avons pas pu mettre en évidence une différence significative entre les deux types d'engrais au seuil de $5 \%$ pour leur effet sur la transpiration spécifique (TS) et la teneur en $\mathrm{K}^{+}$. L'apport de $\mathrm{K}^{+}$sous déficit hydrique, a amélioré la TS chez le génotype $E$ présumé sensible, mais n'a pas eu un effet favorable sur la plupart des autres génotypes. L'effet de l'apport potassique sur l'accumulation des feuilles en ions $\mathrm{K}^{+}$, a varié selon les génotypes et l'humidité du sol. En bref, pour une même fertilité du sol en $\mathrm{K}$, l'absorption $\mathrm{du} \mathrm{K}$ et la transpiration par unité de matière sèche (TS) varient selon les génotypes et l'humidité du sol. 


\section{REFERENCES}

Bonneau X. 1998. Recherches sur les facteurs limitant la production végétale en conditions de stress hydrique. Cas du cocotier à Gunung Batin (Indonésie) : Rôles du chlore dans l'économie de l'eau. Thèse de Doctorat, Institut National Agronomique Paris - Grignon (France), 195 p.

Braconnier S. 1988. Physiologie de la nutrition en chlorure chez le palmier à huile et le cocotier. Thèse de Doctorat. Université des Sciences et techniques du Languedoc Montpellier (France), $207 \mathrm{p}$.

Braconnier S. et A. J. d'Auzac. 1989. Effet d'une carence en chlorure au champ chez le cocotier hybride PB 121. Oléagineux 44 (10) : 468-472.

Braconnier S. et A. J. d'Auzac. 1985. Etude anatomique et mise en évidence cytologique des mouvements de potassium et de chlore associés à l'ouverture des stomates de palmier à huile et de cocotier. Oléagineux 40 (11) : 547-551.

Cornaire B., Daniel C., Zuly-Fodil Y. et E. Lamade. 1994. Le comportement du palmier à huile sous stress hydrique. Données du problème, premiers résultats et voies de recherche. Oléagineux 49 (1) : 1 - 12.

Corrado F. et W. Wuidart. 1990. Germination des graines de palmier à huile (Elaeis guineensis) en sacs de polyéthylène. Méthode par <<chaleur sèche>>. Oléagineux 45 (11) : 511 - 514.

De Taffin G. et R. Ochs. 1973. La fumure potassique du palmier à huile au Dahomey. Oléagineux 28 (6) : 269 - 274

Ollagnier M. 1985. Réactions ioniques et conduite de la fertilisation en liaison avec la résistance à la sécheresse des oléagineux pérennes (palmier à huile et cocotier). Oléagineux 40 (1) : 1 - 7 .

Ollagnier M. et R. Ochs. 1971. Le chlore, nouvel élément essentiel dans la nutrition du palmier à huile. Oléagineux 26 (1) : 1 - 15.

Quencez P. et G. De Taffin. 1981. Relation entre la nutrition potassique et la pluviométrie en culture de palmiers à huile et cocotiers. Oléagineux 36 (1) : 1 - 5 .

Sinclair T. R. et M. M. Ludlow. 1986. Influence of soil water supply on the plant water balance of four tropical grain legums. Australia J. plant Physiology $13: 329$ - 341.

Soltner D. 1994. Les bases de la production végétale. Tome1. Le sol. Collection Sciences et Techniques Agricoles, Le Clos Lorellel. $20^{\mathrm{e}}$ éd . $467 \mathrm{p}$. 